\title{
Improve the Resilience of Multilayer Supply Chain Networks
}

\author{
Hui Xia 1 \\ Hunan Radio and Television University, Changsha 410004, China \\ Correspondence should be addressed to Hui Xia; xiahy111@163.com \\ Received 5 September 2019; Accepted 27 December 2019; Published 17 January 2020 \\ Academic Editor: Honglei Xu \\ Copyright (c) 2020 Hui Xia. This is an open access article distributed under the Creative Commons Attribution License, which \\ permits unrestricted use, distribution, and reproduction in any medium, provided the original work is properly cited. \\ Due to the increasingly complex and dynamic features of global supply chain networks, it is challenging to provide high supply \\ availability and network connectivity under unexpected disruptions. In this paper, we investigate how to improve the topology \\ resilience of the supply chain network from its multilayer nature. We firstly conduct the study on the connectedness in the supply \\ chain network from a topological perspective and adopt the $t$-core method to decompose the network into multiple layers. Then, \\ we propose a layer-based rewiring algorithm to recover the network from disruptions. The experimental results in the real supply \\ chain network show that our design greatly improves the network resilience under both random and targeted disruptions.
}

\section{Introduction}

Nowadays, the supply chain network is becoming increasingly vulnerable in the dynamic and complex business environment. These disruptions largely come from unexpected events such as supplier bankruptcies, natural disasters, and even terrorism attack. One disruption not only disables a few entities in the local supply system but also propagates or even amplifies its impact on the global interconnected systems [1-3]. Therefore, how to improve the resilience of complex supply chain networks is very important in the current global supply system $[4,5]$.

A multitude of risk management strategies and technologies have been proposed to identify, assess, and resolve disruption problems from the perspective of supply network topology [6]. Studies on the supply network topology enable the researcher to quantify complexity and understand the rationale behind the structure and self-organization of the supply chain network in the current business system. Despite much recent progress in analyzing, constructing, and recovering the topology of the supply chain network, how to improve the topology resilience from its multilayer nature has remained elusive.

Supply chain networks represent a graph of nodes and connections, which transfer goods from suppliers to customers. The entities in a complex supply chain network may play various roles and have different impacts on the network resilience. For example, upon removing a few strongly connected core nodes from the supply chain network, the periphery nodes connects to the core ones may lose their connections to the whole network. On the contrary, the failure of one periphery node may only affect the connection to its upstream node, without catastrophic impact on the whole supply system. In this sense, as opposed to conventional homogeneous networks, the supply chain networks have heterogeneous nodes, which could be classified into different layers according to their impacts on network resilience. Intuitively, the nodes could be classified according to their assigned roles. However, current elastic supply chain networks such as the military logistic network may adaptively change the node role and transfer direction according to real-time requirements [7]. Therefore, only using assigned roles to classify nodes potentially leads to wrong classification results in highly complex and dynamic network scenarios.

In this paper, we investigate how to improve the resilience of complex and heterogeneous supply chain networks from a topological perspective. Specifically, we firstly study and analyze the connectedness in the real supply chain network. Then, we adopt the $t$-core decomposition method [8] to distinguish the layers of the network. That is, all nodes in the supply chain network are classified into multiple layers 
based on their impacts on network resilience. Finally, based on the different layers of each node in the multilayer supply chain network, we propose a new rewiring algorithm to recover from the disruptions. The test results show that our design greatly improves the network robustness on supply availability and network connectivity in presence of both random and targeted disruptions.

The remainder of the paper is organized as follows. We first present the related works on the resilience of the supply chain network in Section 2. In Section 3, we conduct an extensive study to explore the impacts of disruptions on network resilience in a typical heterogeneous supply chain network. In Section 4, we show how to use the $t$-core decomposition method to distinguish layers in the supply chain network. We introduce the rewiring algorithm to recover the network from the disruptions in Section 5. In Sections 6 and 7, we show the test results of the military logistic network. Finally, we give the conclusion remarks and future research in Section 8.

\section{Related Work}

The modern supply chain network are confronted with dynamic and unpredictable disruption risks with short or long term negative effects on the network performance $[9,10]$. To recover the network after disruptions, the dynamic adjustments and resource reallocation are needed to ensure the system resiliency $[11,12]$.

After discovering a disruption, the network manager should immediately alleviate the disruption impact, handle ripple effects, and restore network functions. Specifically, to reduce the impact of disruption in the postdisruption stage, many proactive risk management strategies including robust strategy and redundancy strategy are employed in advance $[13,14]$. For example, the backup suppliers, multiple sourcing, and rerouting strategies are used before and after disruptions to mitigate the disruption impact [15-17]. Moreover, immediate and effective handling of ripple effect is also needed to limit the impact of disruption through multiple echelons $[18,19]$. To handle the disruption, these proactive risk management strategies make mitigation and contingency plans in advance to certain disruption events. Unfortunately, due to the highly dynamic feature of the supply chain network, it is hard to make contingency plans suitable for all unexpected disruptions [20].

Research on complex networks has revealed that the real-world networks, such as the Internet and social networks, often have complex topologies that are different from lattice or random graph structures [21]. The topological study is also introduced into research of the resilience of supply chain networks [7]. The results show that the hierarchical supply chain networks are easily disconnected under disruptions.

To improve the robustness of the topology of hierarchical supply chains networks, hierarchy + growth model extends hierarchical supply chains networks by connecting the edges between nodes of the same type [22]. A probabilistic and localized rewiring approach is proposed to improve resilience [23] by probabilistically disconnecting edge from high-degree node and randomly reconnecting to the other node. Consequently, the hierarchical supply chain network eventually evolves into a scale-free network with high resilience. Considering the heterogeneous roles of nodes, the hybrid and tunable network growth model DLA extends the hierarchy + growth model by allowing new nodes to make connections according to both degree and locality [24]. The study of self-organization characteristic in the cluster supply chain network reveal that the key to resilient recovery lies in the local self-organization repair behavior of old and new nodes [25]. Based on the heterogeneous feature of the supply chain network, a resilient SC growth (RSCG) model [26] is established by using the preferential attachment (i.e., each node preferentially attaches based on its unique degree and characteristics).

In contrast with the above study on the supply chain network, we analyze and recover the topology through a new perspective and improve the topology resilience from its multilayer nature, which has remained elusive from the recent research. We adopt the $t$-core decomposition to distinguish the layers of the network according to the node impact and propose the layer-based rewiring algorithm to recover from disruptions. With the consideration of multilayer nature of the supply chain network, our design successfully improves the network resilience on supply availability and network connectivity.

\section{Connectedness Analysis}

In this section, we analyze the characters of a typical supply chain network in the real world. We use the topological metrics to investigate the relationship between nodes and give the insight into connectedness of the supply chain network.

3.1. Evaluation Settings and Metrics. We use the data set collected from the supply chain network of transportation of freight and cargo [27], which consists of 626 nodes and 1112 edges. The nodes represent the source and intermediate and destination locations in the transportation of freight and cargo. The edges are the transportation paths between pairs of nodes. We count the number of goods on the edges during different given time periods and use it as the weight of edge. In this test, we use the topological metrics of average path length, clustering coefficient, and distribution of node degree, which are defined as the following.

Average path length (APL): we define APL as the average value of the number of edges in the shortest path between all pairs of nodes.

Average clustering coefficient (ACP): suppose that a node has $k$ neighbors. Then, for the $k$ neighbors, there at most exist $k(k-1) / 2$ edges between them (this occurs when each neighbor is connected to every other neighbor). We use the clustering coefficient to denote the fraction of these actually existing edges in the $k(k-$ $1) / 2$ edges. Then, we define the average clustering coefficient as the average of clustering coefficient over all nodes. 
Node degree (ND): for each node, its node degree is the number of neighbors. If the node degree is large, it is strongly connected to the other nodes.

3.2. Results. Firstly, we measure the average path length of the transportation paths between pairs of nodes. Figure 1 shows the measurement results with different time periods.

As shown in Figure 1(a), when the measurement period is less than 5 days, the average path length of the transportation paths shows some deviations. However, when the measurement period is large enough (i.e., $>5$ days), the measurement result converges to 3.55 . We also measure the network diameter, which is the largest path length of transportation paths between pairs of nodes. As shown in Figure 1(b), the network diameter becomes 11 when we collect enough data traces. This results mean that the transportation could be finished within 4 hops at average between any pairs of nodes even when the network diameter is as large as 11 .

Next, we calculate the average clustering coefficient, which reflects the cliquishness of network. To get an accurate result, we set the measurement period as 10 days. The average clustering coefficient of all nodes is 0.39 , which is much larger than a random network with the same network scale. Since both the average clustering coefficient and average path length is small, the supply chain network of transportation of freight and cargo shows the features of the small world network.

Finally, we plot the cumulative distribution function (CDF) of degrees of all nodes in Figure 2. A node with more degree has more connections with the other nodes and larger impact on network resilience. As shown in Figure 2, the degree of $80 \%$ nodes is less than 2 , while less than $5 \%$ nodes has their degrees larger than 4, exhibiting a heavy-tailed distribution. This result reflects the fact that, in the supply chain network of transportation of freight and cargo, the goods are firstly transferred to a few strongly connected nodes and then distributed to a large number of downstream nodes.

\section{Decomposition of Multilayer Supply Chain Network}

Based on the connectedness of the abovementioned typical supply chain network, we observe that it shows the features of the small world and has the heavy-tailed distribution of node degree. In this section, we further use the $t$-core decomposition method [8] to distinguish the layers of network.

The steps of the $t$-core decomposition algorithm include

(1) Firstly, the nodes with degree $=1$ are removed from the network and included in the 1st layer. Moreover, if some nodes become completely disconnected from the main network after the abovementioned removing operations, these nodes are also included in the 1st layer.

(2) In the next iterations, all nodes with degree $=t$ $(t=2,3, \ldots, \max )$ are removed from the network.
Here, max is the maximum degree of all nodes. In step $t$, if the degree of a node is less than $t$ after the removal, the node is also removed in the current iteration. In each iteration, the removed nodes form the corresponding layer.

(3) When all nodes are removed from the network, the decomposition algorithm stops in iteration $t_{\max }$. Nodes removed in iteration $t_{\max }$ are included in the last layer.

We use the $t$ - $t_{\text {core }}$ decomposition method to distinguish the layers in the supply chain network of transportation of freight and cargo. The network is decomposed into 10 layers. However, it is hard to analyze a complex network with too many layers. Thus, we merge some layers according to the average clustering coefficient of nodes.

As shown in Table 1, since the average clustering coefficient of nodes in layer $1 \sim 3$ are very close, we merge the layers 1, 2, and 3 into one layer. Similarly, we also merge layer $4 \sim 5$, layer $6 \sim 7$, and layer $8 \sim 10$ into three layers, respectively. Finally, the original 10 layers are merged into 4 layers, making it easier to analyze the supply chain network.

Figure 3 shows the decomposition result of the supply chain network of transportation of freight and cargo. In each layer, the nodes have the close clustering coefficients. With the increasing layer number, the nodes have larger clustering coefficient and thus have greater impact on the other nodes.

\section{Rewiring Approach for Multilayer Supply Chain Networks}

A resilient supply chain network should be adaptive to disruptions and be able to effectively recover from disruptions. In this section, we present the rewiring approach, which improves the network resilience according to the multilayer feature of supply chain networks.

To improve the resilience of supply chain networks, we add randomness into the network topology in a controlled way. Algorithm 1 shows the pseudocode for rewiring a network after node failure.

When a node fails, we rewire the failure edges between the failed node and its direct neighbors as the following steps. Firstly, the failure edges are removed from the edge set $E$. To avoid being isolated from the network, the other endpoint of the rewired edge will rewire the edge to connect with another new node within a radius $d_{\max }$.

To reduce the cliquishness, we randomly choose a new node in the layer with the smallest layer number. That is, the rewired edge will connect to a node with low clustering coefficient. Consequently, the connectedness in rebuilt network becomes more balanced, improving the resilience of the supply chain network under unexpected disruptions.

Moreover, the maximum rewiring radius $d_{\max }$ gives a distance constraint for rewiring. In the real world, it is more economical to connect two nodes that are closer to each other. Therefore, we set the upper limit on the rewiring radius, which can be either topological distance (i.e., in number of hops) or physical distance. 


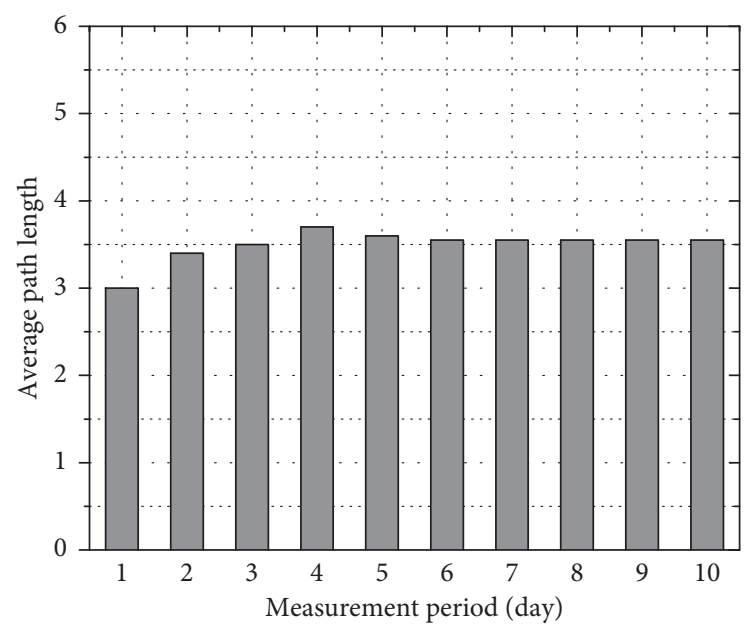

(a)

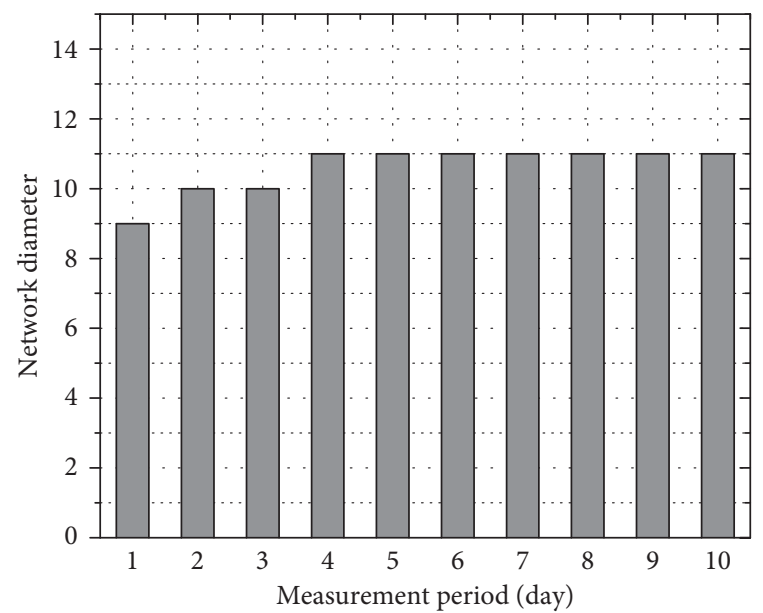

(b)

Figure 1: Path length. (a) Average path length. (b) Network diameter.

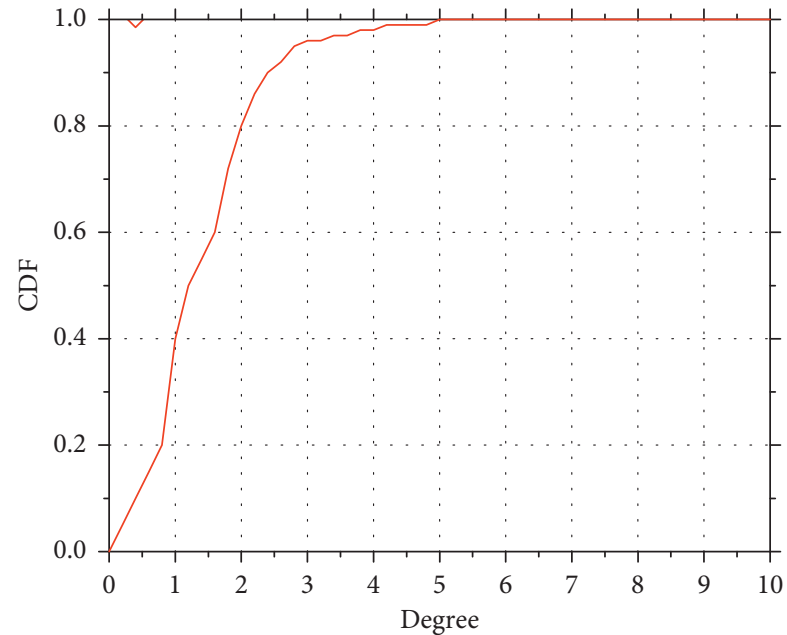

Figure 2: Cumulative distribution function of node degree.

\section{Robustness Evaluation under Disruptions}

In this section, we analyze how a disruption affects the robustness of the supply chain network with multiple layers. Firstly, we describe the evaluation metrics for network robustness. Then, we test the network robustness on supply availability and network connectivity in presence of random and targeted disruptions.

6.1. Evaluation Metric. The modern supply chain network has complex structure and dynamic environment. It is very important to evaluate the network robustness, which represents its ability to maintain function and connectedness once some nodes or edges are lost. A resilient supply chain network is able to maintain the delivery of supplies in response to demands under unexpected disruptions. We use the following metrics to evaluate the network robustness, network efficiency, and impacts of disruption in different layers on the whole network performance.
6.1.1. Network Connectivity. We use the size of the largest connected component to evaluate the network connectivity. In a largest connected component (LCC), there is a path between any pair of nodes [28]. After disruptions occur, for a well-connected network, the size of the largest connected component should still be close to the original network size. Therefore, we use the normalized size of the largest connected component NS to assess the connectivity of the whole network. That is,

$$
\mathrm{NS}=\frac{N^{*}}{N}
$$

where $N^{*}$ is the number of nodes in the largest connected component after disruption and $N$ is the number of nodes in the network before disruption. The value of $N S \in[0,1]$ represents the scale of network connectivity after disruptions occur. The supply chain network with a larger NS has better connectivity and robustness.

6.1.2. Network Efficiency. In the high effective supply chain network, the goods should be transferred within a very short distance. Though the size of largest connected component indicates the network connectivity after disruptions, it cannot directly show the network efficiency. Therefore, we define the network efficiency $E$ as

$$
E=\frac{2}{N(N-1)} \sum_{i \neq j \in V} \frac{1}{d_{i j}}
$$

where $N$ is the number of nodes in the whole network and $d_{i j}$ is the length of the shortest path between node $v_{i}$ and node $v_{j}$. It there is no path between node $v_{i}$ and node $v_{j}, d_{i j}$ equals infinity and then $1 / d_{i j}$ equals 0 .

We define the normalized network efficiency NE as

$$
\mathrm{NE}=\frac{E^{*}}{E}
$$

where $E^{*}$ and $E$ are the network efficiency after and before disruption, respectively. 
TABLE 1: Layers of supply chain network of transportation of freight and cargo.

\begin{tabular}{|c|c|c|c|}
\hline Unmerged layer & Average clustering coefficient & Merged layer & Number of nodes \\
\hline 1 & 0.08 & \multirow{3}{*}{1} & \multirow{3}{*}{142} \\
\hline 2 & 0.125 & & \\
\hline 3 & 0.152 & & \\
\hline 4 & 0.291 & \multirow{2}{*}{2} & \multirow{2}{*}{416} \\
\hline 5 & 0.312 & & \\
\hline 6 & 0.62 & \multirow{2}{*}{3} & \multirow{2}{*}{50} \\
\hline 7 & 0.57 & & \\
\hline 8 & 0.82 & \multirow{3}{*}{4} & \multirow{3}{*}{18} \\
\hline 9 & 0.81 & & \\
\hline 10 & 0.89 & & \\
\hline
\end{tabular}

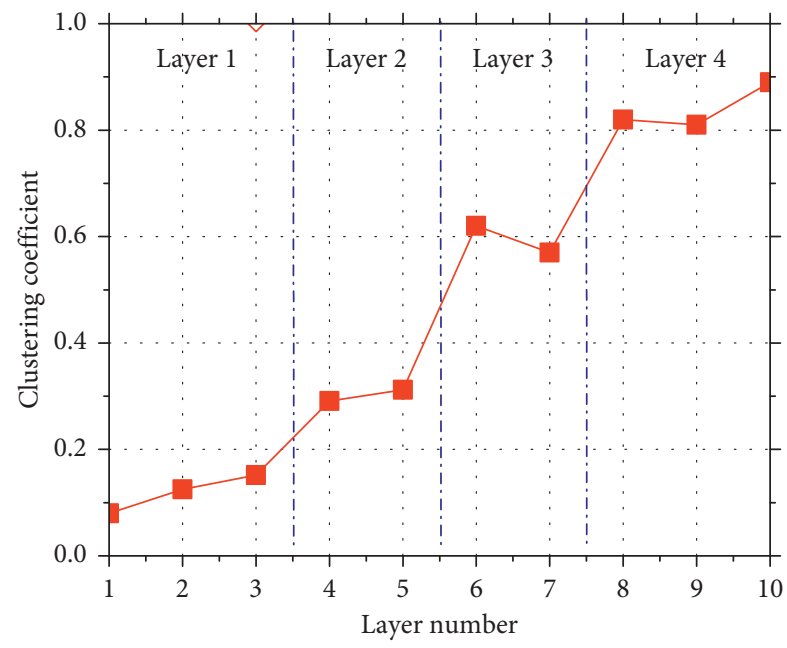

Figure 3: Decomposition result.

6.1.3. Variation Ratio of Robustness. In the multilayer supply chain network, the nodes in different layers have different impacts on the network robustness. To assess the impact of disruption in different layers, we define the variation ratio of network connectivity as

$$
\Delta S=\frac{\mathrm{NS}_{1}-\mathrm{NS}_{2}}{\Delta N}
$$

where $\mathrm{NS}_{1}$ and $\mathrm{NS}_{2}$ are the normalized sizes of the largest connected component after two different disruptions, respectively, and $\Delta N$ is the difference between numbers of failure nodes in two disruptions.

Similarly, we define the variation ratio of network efficiency as

$$
\Delta E=\frac{\mathrm{NE}_{1}-\mathrm{NE}_{2}}{\Delta N}
$$

where $\mathrm{NE}_{1}$ and $\mathrm{NE}_{2}$ are the normalized network efficiency after two different disruptions, respectively.

6.2. Test Setting. In this test, we use the supply chain network of transportation of freight and cargo [27] to assess its robustness under random disruptions and targeted disruptions. The nodes in the supply chain network of transportation of freight and cargo are already classified into 4 layers.
We removed a set of nodes from the network to simulate two types of network disruption including random and targeted disruptions. (1) Random disruptions: in order to simulate random disruptions, we progressively remove a randomly selected set of nodes from the whole supply chain network. (2) Targeted disruptions: for the targeted disruptions, we select the removed nodes in the certain layer to test the impact of different layers.

\section{Results}

We measure the resilience metrics including network connectivity, network efficiency, and variation ratio of robustness after disruptions to evaluate and compare the network connectivity and efficiency under random disruptions and targeted disruptions.

7.1. Network Connectivity and Efficiency under Random Disruption. We measure the network connectivity and efficiency under random disruption. We increase the ratio of failure nodes from 0 to $100 \%$. As shown in Figure 4, when the ratio of failure nodes increases, both network connectivity and efficiency are reduced. Moreover, the decreasing speed of network efficiency is faster than that of network connectivity. This result shows that, the nodes have multiple optional paths to keep good network connectivity under random disruption. However, since the optional paths are usually larger than the failure path, the network efficiency is unavoidably degraded.

7.2. Network Connectivity under Targeted Disruption. We measure the normalized size of the largest connected component with the increasing ratio of failure nodes in different layers. Figure 5(a) shows that, when targeted disruptions happen in all layers, the network connectivity decreases. The disruptions in layers with larger layer number have greater impact on the network connectivity due to the higher clustering coefficient of nodes in these layers. Figure 5(b) shows the variation ratio of network connectivity. In all layers, layer 4 has the greatest impact on the network connectivity, meaning the nodes in layer 4 are more important than the other nodes in ensuring network connectivity.

7.3. Network Efficiency under Targeted Disruption. We measure the network efficiency when the targeted 
Initialization:

$E \longleftarrow$ all edges;

$V \longleftarrow$ all nodes;

On detecting the failure of $v_{f}$ :

begin

For each $v_{r} \in$ direct neighbors of $v_{f}$ do

begin

$E=E-\left\langle v_{r}, v_{f}\right\rangle ;$

Identify $V_{c} \in V$, such that $\forall v_{j} \in V_{c}, 0<\operatorname{distance}\left(v_{r}, v_{j}\right)<d_{\max }$;

$v_{\text {new }}=\operatorname{Random}\left(v_{j} \in\right.$ the nodes with the smallest layer number in $\left.V_{c}\right)$;

If $v_{\text {new }} \notin$ direct neighbors of $v_{r}$

begin

$E=E+\left\langle v_{r}, v_{\text {new }}\right\rangle$

Algorithm 1: Rewiring algorithm.

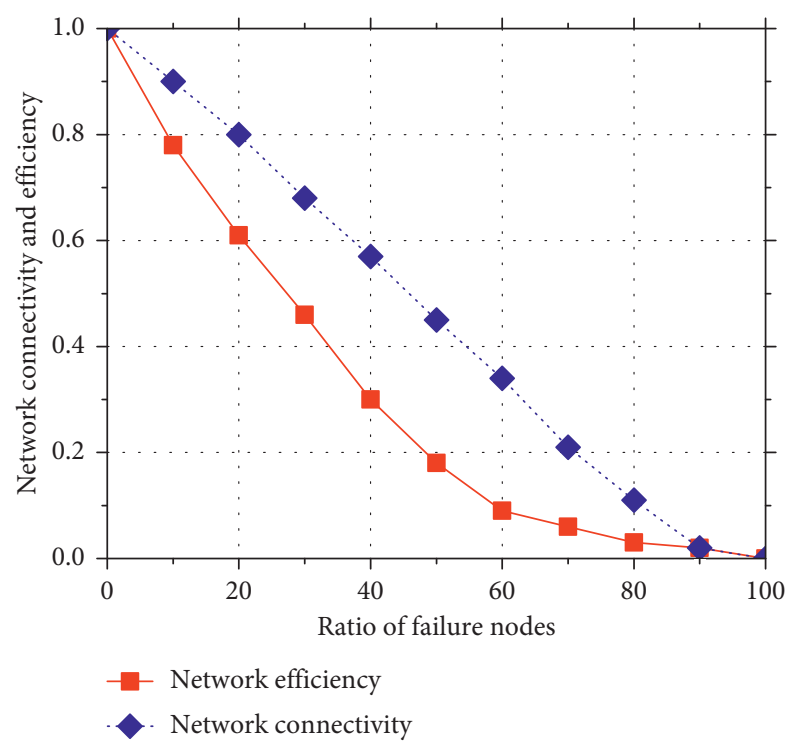

FIgURE 4: Network connectivity and efficiency.

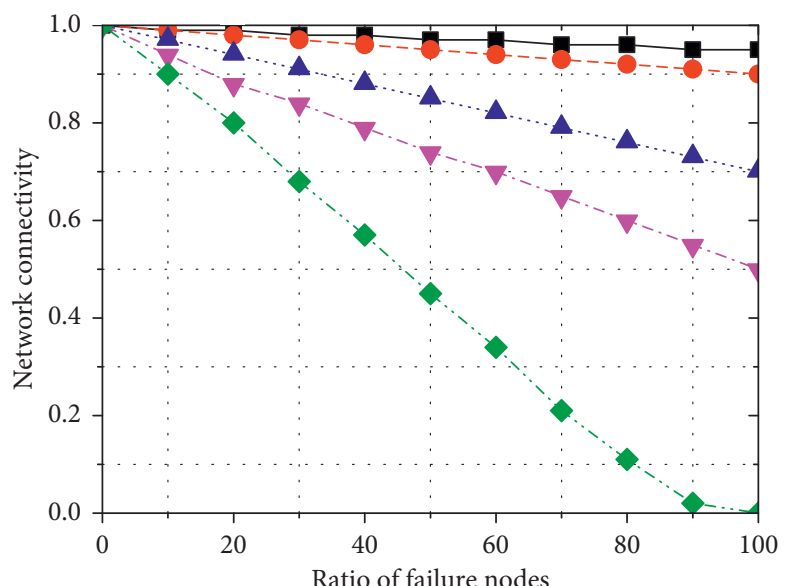

$-\boldsymbol{\nabla}$. Layer 4

๑. Whole

(a)

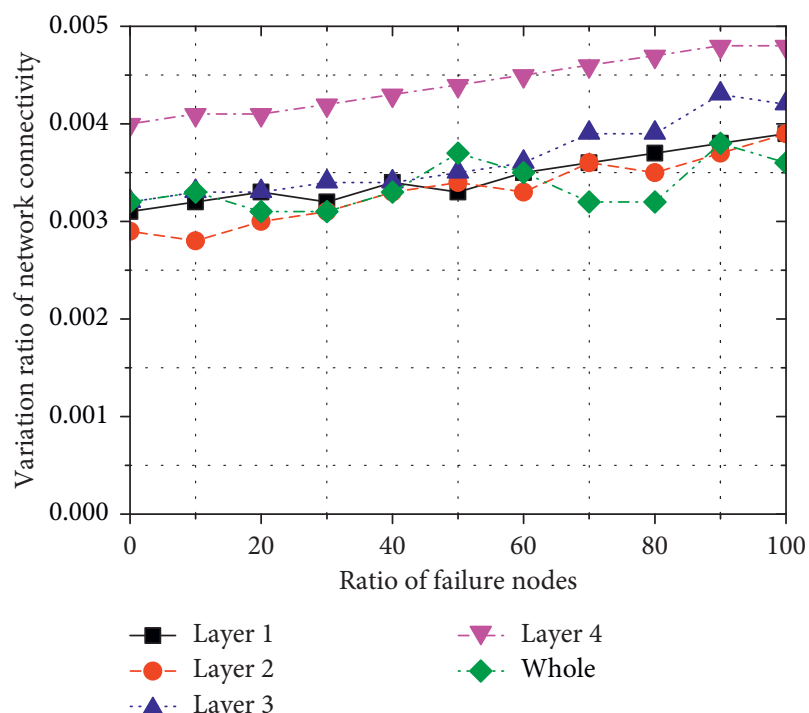

(b)

FIgURE 5: Network connectivity under targeted disruption. (a) Network connectivity. (b) Variation ratio of network connectivity. 


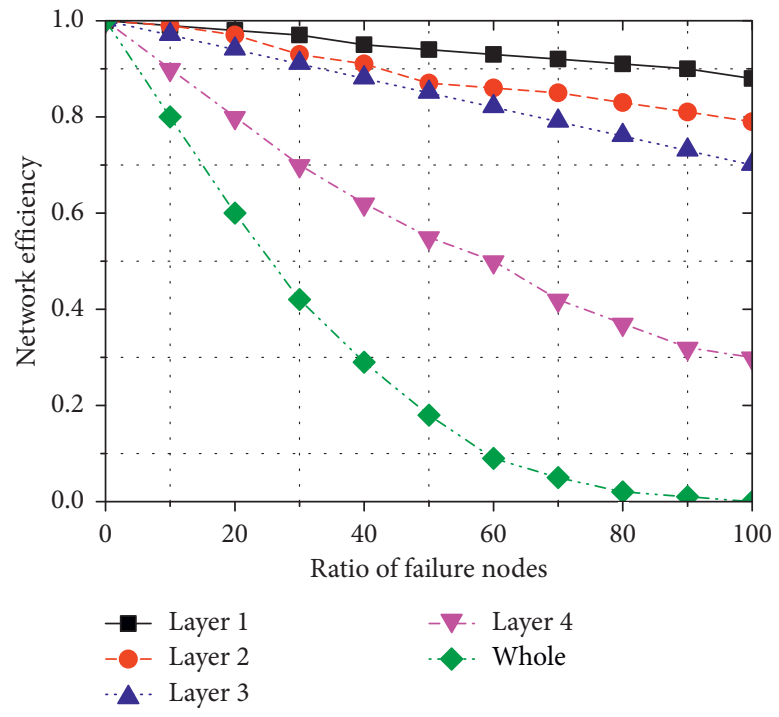

(a)

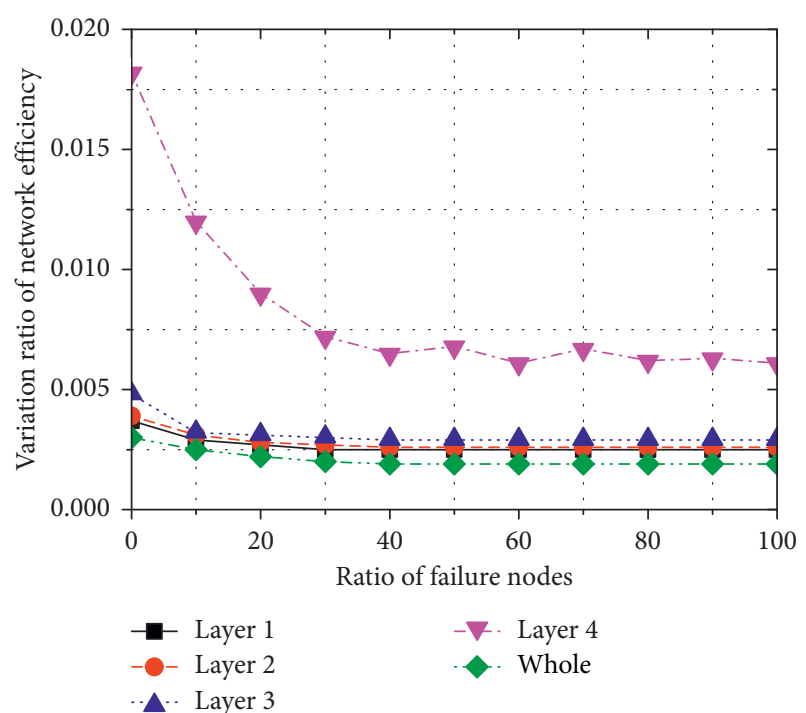

(b)

Figure 6: Network efficiency under targeted disruption. (a) Network efficiency. (b) Variation ratio of network efficiency.

disruptions happen in different layers. Figure 6(a) shows that, with more failure nodes, the network efficiency is reduced. The targeted disruptions in layer 4 have the greater impact on network efficiency than the other layers. The reason is that most junction nodes of the shortest paths are in layer 4 . When the targeted disruptions happen in layer 4, the nodes have to reselect the paths other the shortest ones, resulting in lower network efficiency. This result is also verified in Figure 6(b), in which the failure of nodes in layer 4 has the largest variation ratio of network efficiency. Compared with layer 4 , the impact of other layers on network efficiency is much smaller.

\section{Evaluation on Disruption Recovery}

In this section, to evaluate the effectiveness of rewiring algorithm, we conduct the simulation test with a typical military logistic network under random and targeted disruptions. Since the fundamental target of a supply chain network is to deliver supplies from the supplier to the consumer, we use supply availability as a critical robustness metric to test if the consumer can obtain its supplies from the supplier. Specifically, consider a supply chain network with the supplier, consumer, and relay nodes. The two nonoverlapping subsets of the consumer and supply nodes are sets $V_{\mathrm{C}}$ and $V_{\mathrm{S}}$, respectively. We use $V_{\mathrm{C}}^{*}$ to denote the set of consumer nodes that can access to supply nodes through paths in the supply chain network.

We define the supply availability SV as the ratio between the cardinalities of sets $V_{\mathrm{C}}^{*}$ and $V_{\mathrm{C}}$ :

$$
\begin{aligned}
& V_{\mathrm{C}}^{*}=v_{i} \in V_{\mathrm{C}} \mid \exists v_{j} \in V_{\mathrm{S}}: \exists p_{i j}, \\
& S V=\frac{\left|V_{\mathrm{C}}^{*}\right|}{\left|V_{\mathrm{C}}\right|} .
\end{aligned}
$$

We conduct the simulation test in a military logistic network [7], which consists of 1000 nodes, including 150 supply, 550 demand, and 300 relay nodes. According to impact of nodes on network resilience, the military logistic network is divided into 5 layers. The maximum rewiring radius $d_{\max }$ is set as 3 hops. To give a comprehensive performance evaluation, we set different probabilities $p$ to trigger the rewiring operation after disruptions. In the test, we first construct the supply chain network using the military logistic network configuration. Then, we will simulate disruptions and measure network performance using the aforementioned robustness metrics.

Figure 7 shows the network performance under random disruption, in which the nodes are randomly removed from the network. If $p$ is 0 , there is no rewiring operation. As shown in Figure 7(a), the larger supply availability is achieved with the higher rewiring probabilities $p$. Figure 7(b) shows that the rewiring approach obtains much better connectivity compared to the case without rewiring. When the ratio of failure nodes is $40 \%$, the connectivity improvement is up to about 19\%. As shown in Figure 7(c), however, the rewiring achieves better availability and connectivity at the cost of delivery efficiency. With larger rewiring probability $p$, the nodes have more chances to select suboptimal paths, leading to lower network efficiency.

To evaluate the network performance under targeted disruption, we remove nodes in the order of decreasing node layer. In Figure 8(a), the supply availability decreases faster with increasing ratio of failure nodes compared with the case of random disruption. Figure 8(b) shows the similar trend in network connectivity. Nonetheless, the rewiring operation achieves both higher supply availability and lager network connectivity. Figure 8(c) shows the loss of network efficiency due to rewiring. However, compared with the performance gain in network resilience, the efficiency degradation is acceptable. 


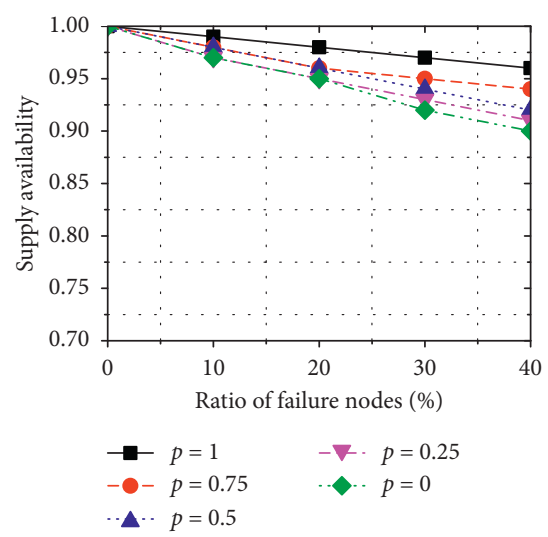

(a)

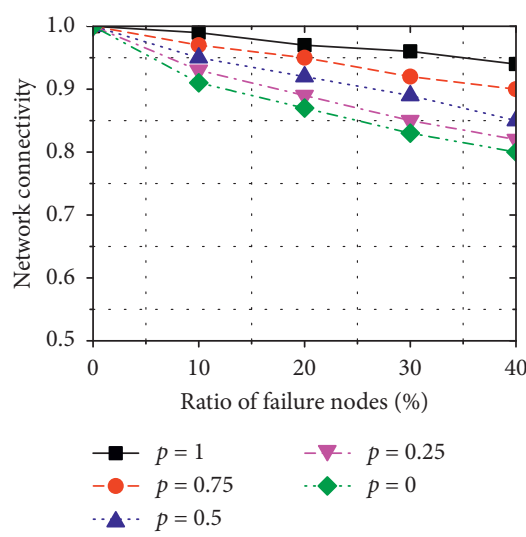

(b)

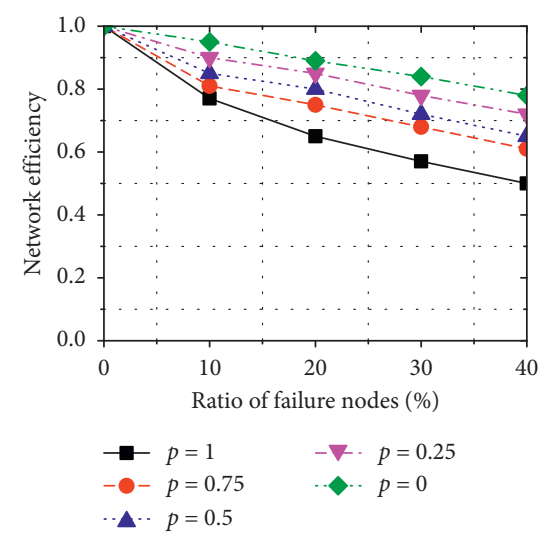

(c)

FIgURE 7: Military Logistic Network under random disruption. (a) Supply availability. (b) Network connectivity. (c) Network efficiency.

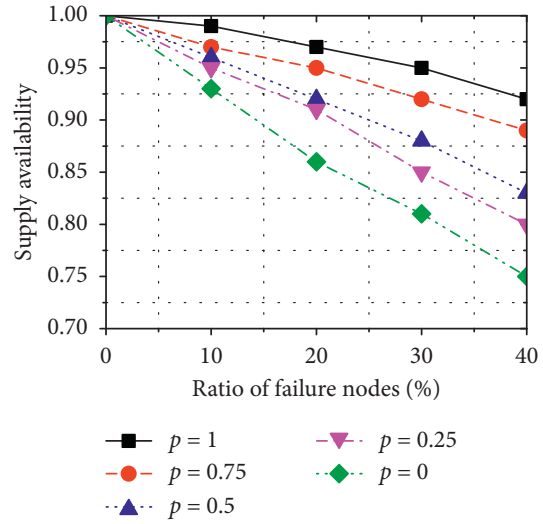

(a)

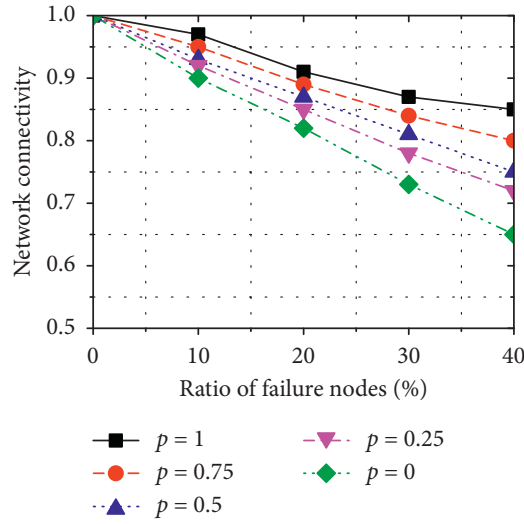

(b)

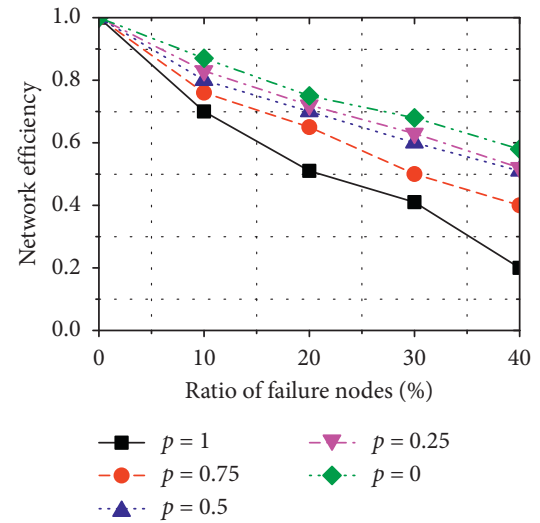

(c)

Figure 8: Military Logistic Network under targeted disruption. (a) Supply availability. (b) Network connectivity. (c) Network efficiency.

\section{Conclusion}

In this paper, we use the complex network theory to investigate how to improve the resilience of the multilayer supply chain networks. Through the topology analysis, we analyze the connectedness in the supply chain network and use the $t$-core algorithm to distinguish the network layers. We also propose a new rewiring algorithm to recover the network from the disruptions. The test results of the real supply chain network show that our design greatly improves the network resilience on supply availability and network connectivity in presence of both random and targeted disruptions.

In future, we would like to address the following issues. Firstly, though we use multiple metrics to analyze the network performance, we will investigate how to combine multiple metrics into a single objective function to evaluate and improve the overall network performance. Secondly, the rewiring operation improves the supply availability and network connectivity, but decreases the network efficiency. We will optimize the probability of rewiring operation to achieve the good tradeoff between the network connectivity and network efficiency.

\section{Data Availability}

The data used to support the findings of this study are available from the corresponding author upon request.

\section{Conflicts of Interest}

The authors declare that they have no conflicts of interest.

\section{Acknowledgments}

This work was supported by the National Natural Science Foundation of China (61872387).

\section{References}

[1] J. Rice and F. Caniato, "Building a secure and resilient supply network," Supply Chain Management Review, vol. 5, pp. 2230, 2003.

[2] S. Chopra and M. S. Sodhi, "Managing risk to avoid supplychain breakdown," MIT Sloan Management Review, vol. 1, pp. 51-63, 2004.

[3] K. B. Hendricks and V. R. Singhal, "An empirical analysis of the effect of supply chain disruptions on long-run stock price 
performance and equity risk of the firm," Production and Operations Management, vol. 1, pp. 35-52, 2005.

[4] P. R. Kleindorfer and G. H. Saad, "Managing disruption risks in supply chains," Production and Operations Management, vol. 1, pp. 53-68, 2005.

[5] T. Wu, J. Blackhurst, and P. O'grady, "Methodology for supply chain disruption analysis," International Journal of Production Research, vol. 45, no. 7, pp. 1665-1682, 2007.

[6] H. Elleuch, E. Dafaoui, A. Elmhamedi, and H. Chabchoub, "Resilience and vulnerability in supply chain: literature review," IFAC-PapersOnLine, vol. 49, no. 12, pp. 1448-1453, 2016.

[7] H. P. Thadakamalla, U. N. Raghavan, S. Kumara, and A. Albert, "Survivability of multiagent-based supply networks: a topological perspective," IEEE Intelligent Systems, vol. 19, no. 5, pp. 24-31, 2004.

[8] S. N. Dorogovtsev, A. V. Goltsev, and J. F. F. Mendes, "K-core organization of complex networks," Physical Review Letters, vol. 4, p. 40601, 2006.

[9] D. Ivanov, "Disruption tails and revival policies: a simulation analysis of supply chain design and production-ordering systems in the recovery and post-disruption periods," Computers and Industrial Engineering, vol. 127, pp. 558-570, 2019.

[10] N. Altay, A. Gunasekaran, R. Dubey, and S. J. Childe, "Agility and resilience as antecedents of supply chain performance under moderating effects of organizational culture within the humanitarian setting: a dynamic capability view," Production Planning and Control, vol. 29, no. 14, pp. 1158-1174, 2018.

[11] D. Ivanov, A. Dolgui, B. Sokolov, and M. Ivanova, "Literature review on disruption recovery in the supply chain," International Journal of Production Research, vol. 55, no. 20, pp. 6158-6174, 2017.

[12] D. Ivanov and A. Dolgui, "Low-Certainty-Need (LCN) supply chains: a new perspective in managing disruption risks and resilience," International Journal of Production Research, vol. 57, no. 15-16, pp. 5119-5136, 2019.

[13] V. Gupta, B. He, and S. P. Sethi, "Contingent sourcing under supply disruption and competition," International Journal of Production Research, vol. 53, no. 10, pp. 3006-3027, 2015.

[14] B. Tomlin, "On the value of mitigation and contingency strategies for managing supply chain disruption risks," Management Science, vol. 52, no. 5, pp. 639-657, 2006.

[15] V. Gupta, B. He, and S. P. Sethi, "Contingent sourcing under supply disruption and competition," International Journal of Production Research, vol. 53, no. 10, pp. 3006-3027, 2015.

[16] T. Sawik, "Integrated selection of suppliers and scheduling of customer orders in the presence of supply chain disruption risks," International Journal of Production Research, vol. 51, no. 23-24, pp. 7006-7022, 2013.

[17] P. Singhal, G. Agarwal, and M. Mittal, "Supply chain risk management: review, classification and future research directions," International Journal of Business Science and Applied Management, vol. 3, pp. 15-42, 2011.

[18] L. Li, Q. Zhang, J. Tian, and H. Wang, "Characterizing information propagation patterns in emergencies: a case study with Yiliang earthquake," International Journal of Information Management, vol. 38, no. 1, pp. 34-41, 2018.

[19] D. Ivanov, "Revealing interfaces of supply chain resilience and sustainability: a simulation study," International Journal of Production Research, vol. 56, no. 10, pp. 3507-3523, 2018.

[20] J. R. Macdonald and T. M. Corsi, "Supply chain disruption management: severe events, recovery, and performance," Journal of Business Logistics, vol. 34, no. 4, pp. 270-288, 2013.
[21] J. Leskovec, L. A. Adamic, and B. A. Huberman, "The dynamics of viral marketing," ACM Transactions on the Web, vol. 1, pp. 142-149, 2007.

[22] H. Thadakamaila, U. N. Raghavan, S. Kumara, and R. Albert, "Survivability of multiagent-based supply networks: a topological perspect," Intelligent Systems, vol. 4, pp. 24-31, 2004.

[23] K. Zhao, A. Kumar, and J. Yen, "Achieving high robustness in supply distribution networks by rewiring," IEEE Transactions on Engineering Management, vol. 58, no. 2, pp. 347-362, 2011.

[24] K. Zhao, A. Kumar, T. P. Harrison, and J. Yen, "Analyzing the resilience of complex supply network topologies against random and targeted disruptions," IEEE Systems Journal, vol. 5, no. 1, pp. 28-39, 2011.

[25] L. Geng, R. Xiao, and S. Xie, "Research on self-organization in resilient recovery of cluster supply chains," Discrete Dynamics in Nature and Society, vol. 2013, Article ID 758967, 11 pages, 2013.

[26] S. I. Mari, Y. H. Lee, M. S. Memon, Y. S. Park, and M. Kim, "Adaptivity of complex network topologies for designing resilient supply chain networks," International Journal of Industrial Engineering, vol. 1, pp. 102-116, 2015.

[27] S. P. Willems, "Real-world multi-echelon supply chains used for inventory optimization: online appendix," Manufacturing and Service Operations Management, vol. 1, pp. 19-23, 2006.

[28] R. Albert, H. Jeong, and A.-L. Barabási, "Error and attack tolerance of complex networks," Nature, vol. 406, no. 6794, pp. 378-382, 2000. 


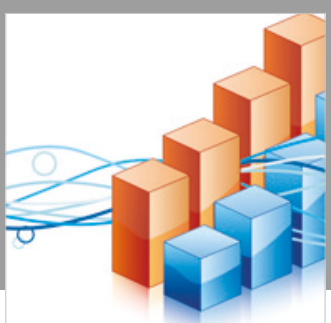

Advances in

Operations Research

\section{-n-m}
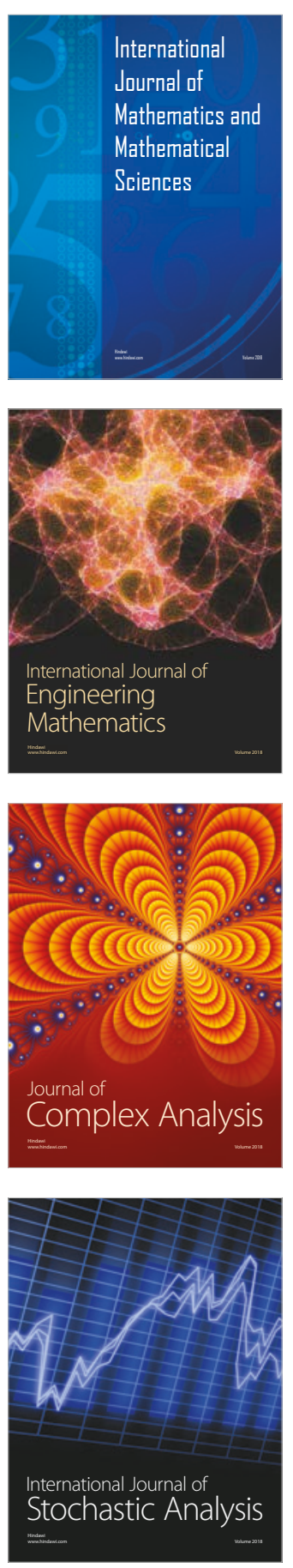
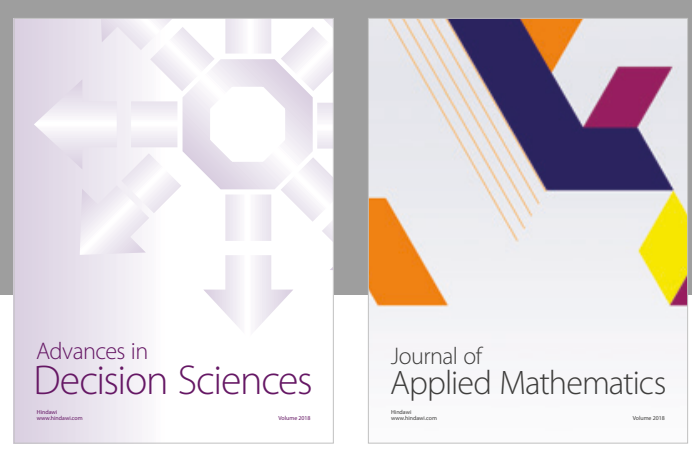

Journal of

Applied Mathematics
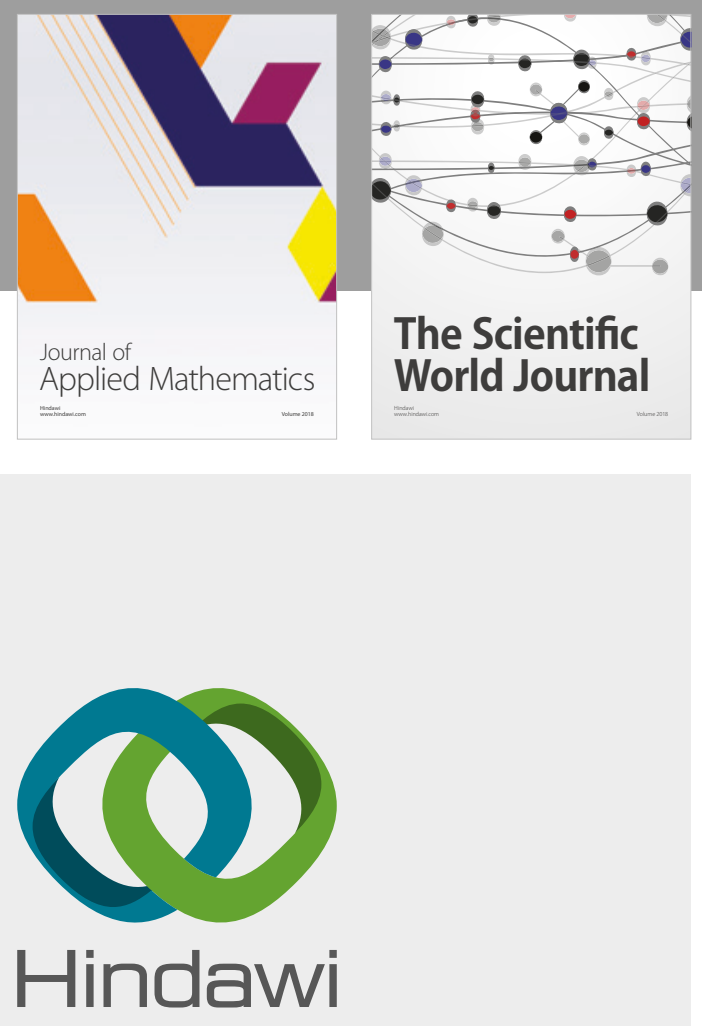

Submit your manuscripts at

www.hindawi.com

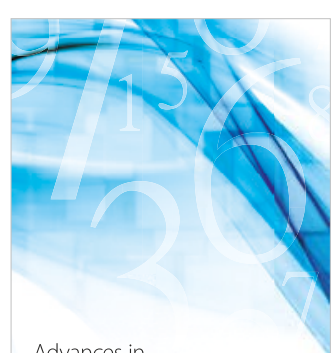

Advances in
Numerical Analysis
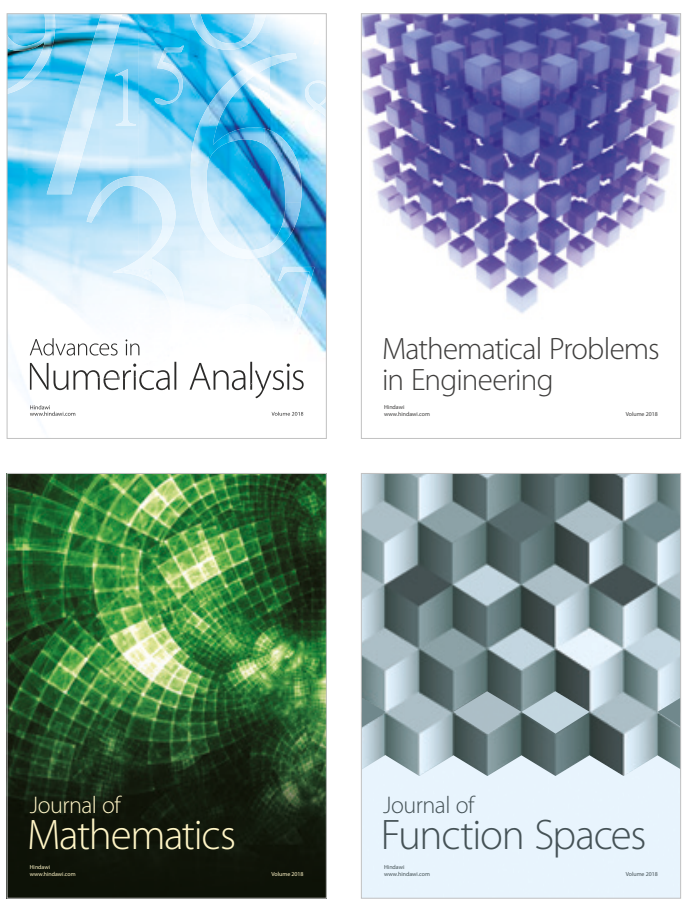

Mathematical Problems in Engineering

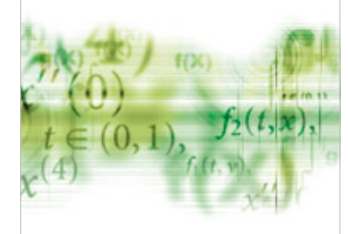

International Journal of

Differential Equations

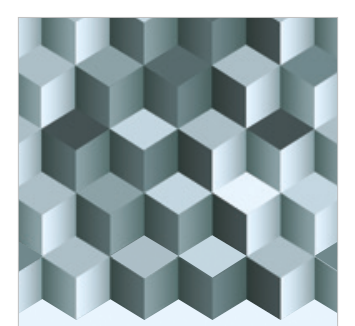

Journal of

Function Spaces
The Scientific

World Journal

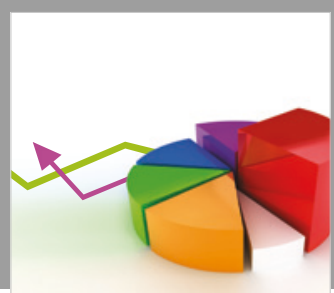

Journal of

Probability and Statistics
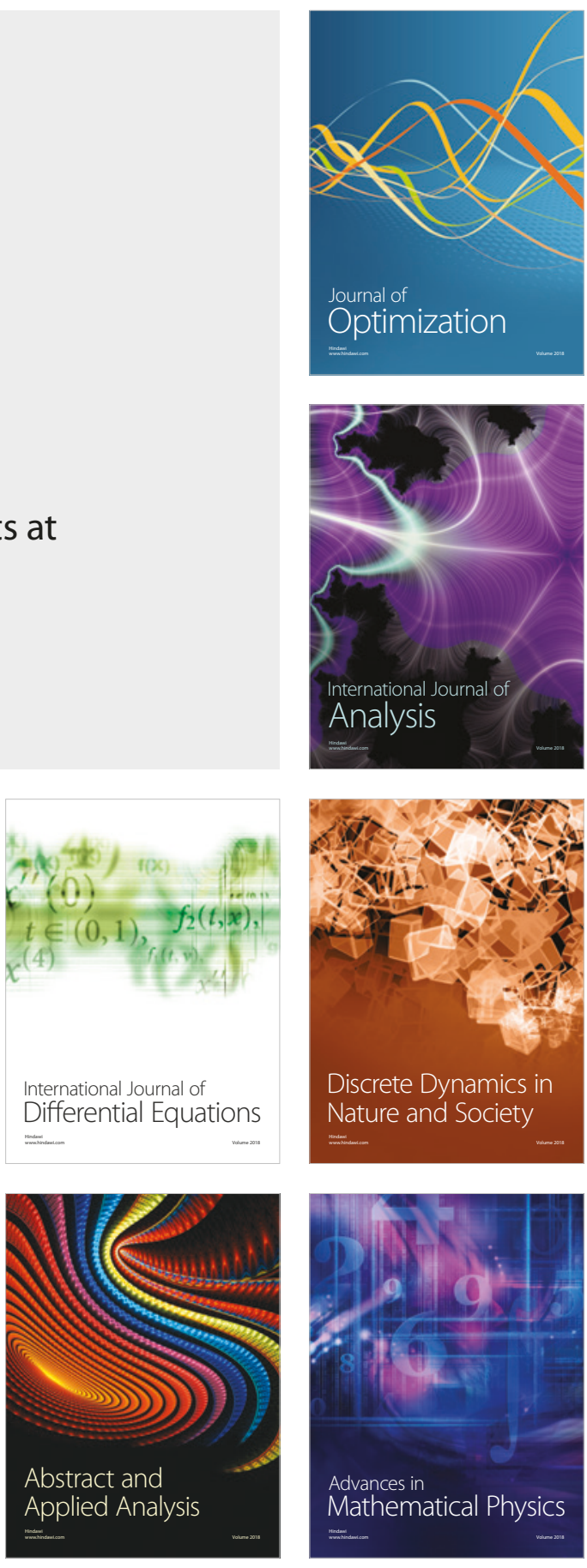